\title{
PERCEPCIONES SOBRE LA ATENCIÓN DE SALUD EN PERSONAS CON PROBLEMAS AUTOIDENTIFICADOS DE SALUD MENTAL EN ZONAS RURALES DEL PERÚ
}

\author{
Javier E. Saavedra ${ }^{1,2, a}$, Verousckha Uchofen-Herrera ${ }^{3, b}$
}

\begin{abstract}
RESUMEN
Una salud centrada en la persona y en las perspectivas de la comunidad acerca de sus necesidades y recursos, es mandatorio en las políticas de atención de salud en contextos de alta diversidad cultural. Desde esta perspectiva, se requiere que la epidemiología se centre no solo en la enfermedad, sino en el diagnóstico de la salud y su contexto, incluyendo las perspectivas de las personas y la comunidad acerca de sus problemas y necesidades. En este artículo se describe y analiza cualitativamente las percepciones de los adultos con problemas de salud mental (PSM) autoidentificados en regiones rurales de la costa sierra y selva del Perú, como factores causales, recursos personales y expectativas de atención de los establecimientos de salud, utilizando el enfoque narrativo de la formulación ideográfica propuesta por la Asociación Mundial de Psiquiatría. Se utilizó la base de datos de los estudios epidemiológicos de salud mental del Instituto Nacional de Salud Mental. El análisis cualitativo de las respuestas de 235 personas revela una notoria adjudicación de los PSM a dinámicas familiares conflictivas y a la pérdida de personas significativas. Se destaca la presencia de escasos recursos comunitarios que ayuden a superar estos problemas. Entre las expectativas de atención de los establecimientos de salud destaca la consejería; sin embargo, un grupo importante de personas desconocen qué esperar del mismo. Consideramos que el enfoque narrativo es una herramienta importante en la perspectiva de una medicina centrada en la persona y la comunidad, y en la planificación de estrategias de intervención.
\end{abstract}

Palabras clave: Medicina centrada en la persona, medicina centrada en la comunidad, narrativas, salud mental, comunidades rurales (fuente: DeCS BIREME)

\section{PERCEPTIONS ON HEALTHCARE IN PEOPLE WITH SELF-IDENTIFIED MENTAL HEALTH PROBLEMS IN THE RURAL AREAS OF PERU}

\begin{abstract}
Person-centered health and community health perspectives on its needs and resources are mandatory in healthcare policies in highly cultural diverse contexts. From this point of view, epidemiology needs to be centered not only on the disease, but also on the health diagnosis and its context, including the points of view of people and the community about their problems and needs. This article describes and qualitatively analyzes the views of adults with self-identified mental health disorders (MHD) in rural regions on the coast, highlands, and jungle of Peru, as causal factors, personal resources, and healthcare expectations from health facilities, using the narrative approach of ideographic formulation proposed by the World Psychiatric Association. The database of mental health epidemiological studies from the National Mental Health Institute was used. The qualitative analysis on answers from 235 people reveals that a large part of MHD is linked to the dynamics of troubled families and to the loss of loved ones. The presence of scarce community resources that help overcome these problems is noted. Counseling is stressed among the expectations of healthcare at facilities; nevertheless, many people do not know what to expect from such healthcare. We believe that the narrative approach is an important tool as regards to community- and person-centered medicine and intervention strategy planning.
\end{abstract}

Key words: Person centered medicine, Community Centered Medicine, narratives, idiographic formulation, rural communities (Source: DeCS BIREME)

\footnotetext{
Instituto Nacional de Salud Mental "Honorio Delgado - Hideyo Noguchi”. Lima, Perú.

Facultad de Medicina, Universidad Peruana Cayetano Heredia. Lima, Perú.

Asociación Ferreycorp. Lima, Perú.

Médico psiquiatra, doctor en Medicina. ${ }^{b}$ Licenciada en Psicología Clínica y magíster en Antropología

Recibido: 21/09/2016 Aprobado: 23/11/2016
}

Citar como: Saavedra JE, Uchofen-Herrera V. Percepciones sobre la atención de salud en personas con problemas autoidentificados de salud mental en zonas rurales del Perú. Rev Peru Med Exp Salud Publica. 2016;33(4):785-93. doi: 10.17843/rpmesp.2016.334.2566 


\section{INTRODUCCIÓN}

Desde el punto de vista de la comunidad, el enfoque de la salud centrada en la persona y la comunidad cumple un papel importante en las políticas de atención de salud debido a que la diversidad cultural de las poblaciones, producida por las migraciones, crea fuentes de inequidad por la emergencia de diferentes necesidades ${ }^{(1,2)}$. La salud centrada en la persona ha sido asociada con temas como valores, atención a los contextos sociales y personales, integración de la salud y el bienestar, participación activa de la persona, la comunicación sensible e interactiva y autonomía (3), a recursos personales, emocionales $e$ instrumentales y comunitarios, además del entendimiento de los puntos y perspectivas de los pacientes acerca de sus problemas ${ }^{(4,5)}$. Este enfoque considera particularidades del paciente en relación a sus síntomas, narrativas ideográficas, el significado de la enfermedad para el paciente, el efecto de las comorbilidades y el ambiente (6). Propuestas formales de diagnósticos integradores que incluyen aproximaciones narrativas o idiográficas han provenido de la Asociación Mundial de Psiquiatría a través de las Guías Internacionales para la Evaluación y Diagnóstico (IGDA) (7) y la Guía Latinoamericana de Diagnóstico Psiquiátrico (GLADP) ${ }^{(8)}$. Este enfoque promueve involucrar más al paciente y su familia en los procesos de cuidados clínicos, y cubrir mejor sus expectativas de salud (9), a través de una formulación diagnóstica que contenga no solo información clínica, sino también una formulación idiográfica (FI) de tipo narrativa que incluya información acerca de perspectivas del paciente y/o la familia acerca del problema clínico.

El objetivo de este artículo es describir y analizar las percepciones que tienen adultos que se han autoidentificado con problemas de salud mental, sobre estos problemas y la atención de salud, en regiones rurales del Perú, es decir las causas adjudicadas a estos problemas, aspectos positivos y recursos personales, recursos comunitarios, y sus expectativas de atención en los establecimientos de salud utilizando el enfoque narrativo de la FI. La zona rural en el Perú es la zona que presenta mayor pobreza, mayor desigualdad y las brechas más altas de atención de salud ${ }^{(10)}$, y donde los hogares dependen en su mayoría de ingresos agrícolas o no diversifican sus actividades económicas, tienen, por lo que son más vulnerables ante eventos negativos inesperados al interior del hogar 0 ante impactos económicos.

\section{METODOLOGÍA}

Estudio descriptivo, transversal, cualitativo utilizando las bases de datos de los estudios epidemiológicos de salud mental realizados por el Instituto Nacional de Salud Mental "Honorio Delgado Hideyo Noguchi"
(INSM) entre el 2007 y 2009 en áreas rurales del Perú del departamento de Lima, y centros poblados rurales próximos a las ciudades de Ayacucho, Cajamarca, Huaraz, Iquitos y Pucallpa.

La población del estudio original comprendió adultos ( $\geq 18$ años) residentes de hogares en las áreas rurales referidas. Las muestras fueron probabilísticas seleccionadas en forma trietápica, por conglomerados, según información del Instituto Nacional de Estadística e Informática. La muestra total obtenida fue de 2536 en Lima, 3031 en la sierra y 2331 adultos en la selva. La tasa total de no respuesta (considerando el efecto de viviendas y personas) fue de $6,1 \%, 5,8 \%$ y $0,3 \%$ respectivamente. Para este estudio se consideraron a las personas que en el momento de la entrevista reportaran estar padeciendo algún problema emocional o de los nervios (morbilidad sentida).

La encuesta fue realizada por profesionales de la salud, principalmente psicólogos con entrenamiento de al menos 70 horas. La calidad de los datos se aseguró a través de reentrevistas aleatorias, supervisión directa, análisis de concordancia supervisor-entrevistadores. Mayor detalle de la metodología y de la operación de campo puede ser encontrado en estudios del Instituto de Salud Mental $(11,12,13)$.

Detalles psicométricos de los instrumentos usados por el INSM han sido publicadas en otra parte ${ }^{(14)}$. La evaluación de las percepciones de las personas sobre sus PSM se basó en la formulación idiográfica personalizada sugeridas en el IGDA y la GLADP $(15,16)$. Esta formulación narrativa se divide en tres secciones: 1) Problemas clínicos y su contextualización, referido a las perspectivas del paciente, la familia y/o el clínico acerca del problema de salud; 2) Factores positivos del paciente pertinentes al tratamiento y promoción de la salud referidos a habilidades personales, recursos sociales, aspiraciones personales y espirituales; y 3) Expectativas sobre restauración y promoción de la salud, referidos a las aspiraciones sobre tratamiento, el estado de salud y calidad de vida en el futuro previsible. Se utilizó el cuestionario de la formulación diagnóstica idiográfica, el cual fue elaborado en estudios previos tomando en cuenta las sugerencias de la FI del IGDA, a través de preguntas abiertas para cada uno de los ejes, las cuales se pusieron a consideración de expertos en el área. El proceso de afinamiento lingüístico de las preguntas incluyó un focus group con personas de la población y un piloto formulando las preguntas a pacientes con problemas psiquiátricos (Tabla 1).

Los trastornos mentales (TM) se estimaron a través del Mini International Neuropsychiatric Interview 
Tabla 1. Cuestionario de la Formulación Idiográfica

\begin{tabular}{l}
\hline Cuestionario \\
\hline I. PROBLEMAS CLíNICOS Y SU CONTEXTUALIZACIÓN \\
1. ¿Cuáles cree usted que son las causas o factores que dieron inicio a su problema de los nervios o de tipo emocional? \\
2. ¿Qué situaciones del pasado han influenciado en su problema de los nervios o de tipo emocional? \\
3. ¿Qué situaciones impiden o no permiten la recuperación de su problema de los nervios o de tipo emocional? \\
II. FACTORES POSITIVOS DEL PACIENTE \\
4. ¿Qué cualidad, fortaleza o recurso personal tiene usted que le ayuda a superar su problema de los nervios o de tipo emocional? \\
5. ¿Qué es lo que le da sentido a su vida? \\
6. ¿Cómo contribuye la fe o su vida espiritual en su salud? \\
7. ¿Qué apoyo ha recibido de su familia o personas cercanas por su problema de los nervios o de tipo emocional? \\
8. ¿Con qué recursos cuenta su familia para ayudarlo(a) en la recuperación de su problema de los nervios o de tipo emocional? \\
9. ¿Con qué recursos cuenta su comunidad para ayudarlo (a) en la recuperación de su problema de los nervios o de tipo emocional? \\
III. EXPECTATIVAS DE RECUPERACIÓN Y PROMOCIÓN DE LA SALUD \\
10. ¿Considera usted que es posible recuperarse del problema de sus nervios o de tipo emocional? \\
11. ¿Qué podría hacer usted para recuperarse del problema de sus nervios o de tipo emocional? \\
12. ¿En qué podrían ayudarlo(a) los demás para recuperarse del problema de sus nervios o de tipo emocional? \\
13. ¿Cómo debería estar usted para considerarse recuperado(a) del problema de sus nervios o de tipo emocional? \\
14. ¿Cómo se ve en el futuro, una vez recuperado(a) del problema de sus nervios o de tipo emocional? \\
15. ¿Qué esperaría usted recibir al ser atendido(a) en un establecimiento de salud por su problema de los nervios o de tipo emocional? \\
16. ¿Qué podría hacer usted para que le vaya mejor en la vida? \\
\hline
\end{tabular}

(MINI-CIE-10) ${ }^{(17)}$, adaptado para obtener prevalencia actual, anual y de vida de los principales trastornos. La morbilidad sentida en el último año se exploró mediante las preguntas: 1) "¿En algún periodo de su vida ha tenido algún problema con sus nervios o de tipo emocional?"; 2) "¿En algún periodo de su vida (más de 7 días) ha tenido... excesiva tristeza, pena o depresión?,...excesivo miedo o pánico o ansiedad?,... ¿ataque de nervios?,... ¿tensión 0 estrés excesivo?,... ¿preocupación excesiva?,... ¿dificultad para relacionarse socialmente con otras personas?,... ¿dificultad para controlar sus pensamientos?,... ¿pensamientos repetitivos $u$ obsesiones?,... ¿problemas con alcohol o drogas?,... ¿excesivo cansancio mental o surmenage?, ... ¿ paranoia o sentir que le perseguían?; 3) ¿Hace cuánto tiempo terminó el último de estos problemas que tuvo?

Se incluyeron datos sociodemográficos y preguntas sobre características de la vivienda, basados en encuestas del INEI (18). Para obtener los indicadores objetivos de pobreza, se utilizó la metodología de las necesidades básicas insatisfechas (NBI) ${ }^{(19)}$. El indicador subjetivo de pobreza se basó en una pregunta formulada a la esposa del jefe del hogar sobre la capacidad de cobertura de las necesidades esenciales o básicas de la familia con los ingresos familiares.

Las prevalencias generales estimadas de la muestra se ponderaron teniendo en cuenta la probabilidad conocida de selección y la restauración de la distribución de la población. Se utilizó el SPSS Ver.19, utilizando la F corregida como variante del estadístico de chi cuadrado corregido de Rao-Scott de segundo orden. El análisis cualitativo fue realizado por uno de los autores (VU), a través del método cualitativo de la Grounded Theory (20, 21). Se procedió a la creación de categorías por cada una de las 16 preguntas del cuestionario, identificándose, clasificándose y codificándose las respuestas atendiendo a criterios temáticos relacionados con los objetivos. Se obtuvo frecuencias, porcentajes y algunas viñetas por cada categoría para ayudar a ejemplificarla mejor.

El protocolo del estudio original fue evaluado y aprobado por el Comité de Ética Institucional. Se solicitó un consentimiento informado escrito antes del inicio de la encuesta.

\section{RESULTADOS}

La muestra final fue de 235 adultos quienes percibieron PSM al momento de la entrevista en las zonas rurales: $41,8 \%$ de la sierra, el $34,5 \%$ de Lima y el $23,7 \%$ de la selva. La edad promedio fue de 46,1 (IC95\%: 42,5$49,7)$. No se encontraron diferencias significativas entre las poblaciones encuestadas en la edad, sexo, estado civil y situación laboral. El analfabetismo y la pobreza subjetiva fueron mayores en el área de la sierra rural. De las personas que percibieron PSM en el momento de la entrevista, un $84,0 \%$ había presentado algún TM alguna vez en su vida, un $69,2 \%$ lo había presentado en el último año y un $67,7 \%$ lo presentaba en el momento de la entrevista, no encontrándose diferencias significativas entre las tres áreas geográficas. (Tabla 2) 
Tabla 2. Características sociodemográficas de la población que percibió problemas de salud mental en el último año en las áreas rurales de Ancash, Ayacucho, Cajamarca, Lima, Loreto y Ucayali $(n=235)$

\begin{tabular}{|c|c|c|c|c|c|}
\hline & $\begin{array}{c}\text { Costa rural = } 81 \\
\%(\text { IC } 95 \%)\end{array}$ & $\begin{array}{c}\text { Sierra rural = } 97 \\
\%(\text { IC } 95 \%)\end{array}$ & $\begin{array}{c}\text { Selva rural = } 57 \\
\%(\text { IC 95\%) }\end{array}$ & $\begin{array}{c}\text { Total } \\
\% \text { (IC 95\%) }\end{array}$ & Valor $p$ \\
\hline Edad promedio (error estándar) & $43,7(3,1)$ & $46,7(2,3)$ & $45,4(2,6)$ & $46,1(1,8)$ & n.s. \\
\hline Grupos de edad & $\%(I C$ 95\%) & $\%(I C$ 95\%) & $\%($ IC 95\%) & $\%(I C$ 95\%) & \\
\hline 18 a 24 & $15,6(5,4-37,6)$ & $12,8(5,4-27,1)$ & $9,0(3,6-20,7)$ & $13,0(6,7-23,8)$ & n.s. \\
\hline 25 a 44 & $42,6(27,6-59,2)$ & $32,1(21,4-45,2)$ & $47,5(36,9-58,4)$ & $35,0(26,0-45,2)$ & \\
\hline 45 a 64 & $26,3(14,2,9-43,4)$ & $40,1(28,4-53,0)$ & $28,6(17,1-43,8)$ & $36,9(27,6-47,3)$ & \\
\hline 65 a más & $15,5(7,7-28,8)$ & $15,0(8,1-26,1)$ & $14,9(7,0-28,9)$ & $15,1(9,3-23,5)$ & \\
\hline \multicolumn{6}{|l|}{ Sexo } \\
\hline Mujer & $60,0(43,1-74,8)$ & $42,2(30,4-55,0)$ & $41,6(27,7-57,0)$ & $45,3(35,5-55,4)$ & n.s. \\
\hline \multicolumn{6}{|l|}{ Nivel educativo } \\
\hline Analfabetismo & $3,8(0,7-17,6)$ & $30,0(21,3-40,4)$ & $11,8(4,2-29,1)$ & $24,2(17,6-32,3)$ & 0,001 \\
\hline Sin nivel & -- & $19,3(12,3-29,0)$ & $9,3(2,9-26,0)$ & $15,2(9,9-22,8)$ & 0,003 \\
\hline Primaria incl. inicial & $34,8(20,8-52,2)$ & $55,2(44,6-65,3)$ & $63,5(45,3-78,6)$ & $52,2(43,3-60,8)$ & \\
\hline Secundaria incl. bach. & $54,0(34,6-72,2)$ & $20,9(11,7-34,5)$ & $24,3(10,7-46,3)$ & $26,9(18,4-37,5)$ & \\
\hline Superior no universitaria & $10,1(4,1-22,5)$ & $4,0(1,5-10,3)$ & $2,9(0,7-11,3)$ & $5,0(2,5-9,8)$ & \\
\hline Superior universitaria & $1,1(0,2-8,2)$ & $0,6(0,1-2,3)$ & -- & $0,7(0,2-2,0)$ & \\
\hline \multicolumn{6}{|l|}{ Estado civil } \\
\hline Conviviente & $32,9(19,1-50,6)$ & $25,5(16,3-37,4)$ & $53,4(36,0-70,1)$ & $28,7(20,9-37,9)$ & 0,071 \\
\hline Separado(a) o divorciado(a) & $7,1(3,3-15,0)$ & $4,6(2,0-10,3)$ & $5,9(2,3-14,6)$ & $5,1(2,8-9,2)$ & \\
\hline Viudo(a) & $10,2(4,1-23,2)$ & $15,1(8,4-25,7)$ & $5,6(2,0-15,0)$ & $13,6(8,2-21,6)$ & \\
\hline Casado(a) & $44,5(28,1-62,1)$ & $34,6(24,8-45,8)$ & $27,0(13,8-46,2)$ & $35,8(27,6-44,9)$ & \\
\hline Soltero(a) & $5,3(1,3-19,3)$ & $20,3(11,6-33,2)$ & $8,0(3,5-17,4)$ & $16,9(10,0-27,1)$ & \\
\hline \multicolumn{6}{|l|}{ Lengua materna } \\
\hline Castellano & $95,4(85,1-98,7)$ & $61,0(50,9-70,3)$ & $85,4(70,6-93,5)$ & $68,7(60,6-75,8)$ & $<0,001$ \\
\hline Quechua & $4,6(1,3-14,9)$ & $39,0(29,7-49,1)$ & $10,9(4,0-26,4)$ & $31,1(24,0-39,2)$ & \\
\hline Otro & -- & - & $3,7(1,0-12,9)$ & $0,2(0,1-0,9)$ & \\
\hline \multicolumn{6}{|l|}{ Condiciones de empleo } \\
\hline Trabajó la semana anterior & $75,2(60,0-86,0)$ & $66,1(51,6-78,1)$ & $62,2(49,5-73,5)$ & $67,4(56,1-77,0)$ & n.s. \\
\hline Desempleo & $2,3(1,8-2,9)$ & $0,3(0,0-2,5)$ & $2,6(0,6-10,1)$ & -- & n.s. \\
\hline \multicolumn{6}{|l|}{ Nivel de pobreza subjetiva* } \\
\hline Pobre extremo & $5,0(1,5-15,9)$ & $8,2(3,3-19,0)$ & $3,0(0,7-12,6)$ & $7,3(3,3-15,3)$ & 0,020 \\
\hline Pobre & $36,7(22,1-54,1)$ & $58,8(44,5-71,7)$ & $42,2(26,8-59,3)$ & $53,8(42,6-64,6)$ & \\
\hline No pobre ajustado & $53,0(32,5-72,6)$ & $33,1(21,7-46,8)$ & $54,7(37,8-70,6)$ & $38,0(28,1-49,1)$ & \\
\hline No pobre & $5,3(0,8-28,6)$ & -- & -- & $0,9(0,1-6,3)$ & \\
\hline \multicolumn{6}{|l|}{ Nivel de pobreza objetiva** } \\
\hline Pobre extremo & $43,2(27,4-60,6)$ & $65,2(51,1-77,1)$ & $73,8(52,1-87,9)$ & $62,0(50,7-72,1)$ & n.s. \\
\hline Pobre & $40,0(24,7-57,5)$ & $29,9(19,0-43,5)$ & $19,8(10,2-35,1)$ & $31,0(21,9-41,7)$ & \\
\hline No pobre & $16,8(7,3-33,8)$ & $4,9(1,3-16,7)$ & $6,4(0,9-33,6)$ & $7,1(3,2-14,8)$ & \\
\hline \multicolumn{6}{|l|}{ Estado de salud mental } \\
\hline $\begin{array}{l}\text { Trastorno mental } \\
\text { (prevalencia de vida) }\end{array}$ & $85,2(69,2-93,6)$ & $84,8(75,0-91,2)$ & $71,5(53,9-84,3)$ & $84,0(76,3-89,5)$ & n.s. \\
\hline $\begin{array}{l}\text { Trastorno mental } \\
\text { (prevalencia anual) }\end{array}$ & $82,1(66,1-91,5)$ & $67,3(56,1-76,9)$ & $57,1(42,0-71,0)$ & $69,2(60,3-77,0)$ & 0,090 \\
\hline $\begin{array}{l}\text { Trastorno mental } \\
\text { (prevalencia actual) }\end{array}$ & $76,4(60,1-87,4)$ & $67,1(55,9-76,7)$ & $52,2(36,0-67,9)$ & $67,7(58,7-75,6)$ & n-s. \\
\hline $\begin{array}{l}\text { Se refiere a la percepción por part } \\
\text { extremo (no cubren ni las necesida } \\
\text { (cubre las necesidades básicas de }\end{array}$ & ésposa del jefe del & $\begin{array}{l}\text { sobre la capacidad } \\
\text { obre (cubren las nec } \\
\text { ); no pobre (cubren I }\end{array}$ & $\begin{array}{l}\text { ertura de las neces } \\
\text { des básicas de alim }\end{array}$ & $\begin{array}{l}\text { des con los ingresos } f \\
\text { ación, pero no otras); } \\
\text { mbién otras como pas }\end{array}$ & $\begin{array}{l}\text { iliares: Pobre } \\
\text { pobre básico } \\
\text { mpos). }\end{array}$ \\
\hline ** Se refiere a la pobreza según cara & sticas objetivables de & encia de necesida & sicas insatisfechas & I) como condicion & la vivie \\
\hline
\end{tabular}




\section{ANÁLISIS DE LAS PREGUNTAS DE LA FORMULACIÓN IDIOGRÁFICA}

Se presentan los resultados derivados de las encuestas narrativas que constituyen las percepciones sobre los PSM, organizados en las siguientes categorías con algunas viñetas: 1) Causas de los problemas de tipo emocional; 2) Recursos para afrontar los problemas de tipo emocional: Personales, familiares y comunitarios; y 3) Expectativas de atención en los establecimientos de salud.

\section{CAUSAS DE LOS PROBLEMAS DE TIPO EMOCIONAL}

Un $24,6 \%$ de las causas de los PSM según la perspectiva de los entrevistados se relaciona con problemas familiares o conyugales, como malas relaciones familiares, infidelidad, violencia, abandono, maltrato, y problemas cotidianos con los hijos. Cabe mencionar que el conflicto conyugal es más mencionado por las mujeres $(21,3 \%)$ que los hombres $(5,9 \%)$ (Tabla 3$)$.

¿Cuáles cree usted que son las causas o factores que dieron inicio a su problema de los nervios o de tipo emocional?

$\sqrt{ }$ Mi pareja tuvo otra pareja y temo que me vaya a dejar (...) Tengo una amiga y así le pasó, el hombre tenía su compromiso, se fue y la dejó (mujer,20 años, Ayacucho).

$\sqrt{ }$ El maltrato sufrido, me sentía algo triste por los problemas de mis padres, me sentía asustado (...) Los castigos que me daban que me amarraban en un tronco y me pegaba mi padrastro, me empezaba a pegar (hombre, 26 años, Loreto).

Otras de las causas mencionadas de PSM fue la muerte de una persona significativa $(15,7 \%)$, ya sea la pareja o cónyuge, o familiar cercano y lo que suele afectar la economía del hogar, se muestra en las siguientes viñetas:

¿Cuáles cree usted que son las causas o factores que dieron inicio a su problema de los nervios o de tipo emocional?

$\sqrt{ }$ La muerte de mi esposa con cáncer al útero (hombre, 65 años, Cajamarca).

$\checkmark$ La muerte de mi esposo y mi hijo (...) Quedarme sola con los problemas (...) La preocupación de dinero, que mi hijo no estudia, no hay trabajo (mujer, 59 años, Cajamarca).

El estresor social $(10,6 \%)$ como factor causal tiene que ver con situaciones como robos, disputa por terrenos, disputa entre miembros de la comunidad, huelga de mineros, violencia, influencia de amigos, migración laboral. En cuarto lugar, aparece la salud como causa de los PSM con 10,2\%.
¿Cuáles cree usted que son las causas o factores que dieron inicio a su problema de los nervios o de tipo emocional?

$\sqrt{ }$ Mucha preocupación por motivo de los mineros (...) Mucha preocupación por los cultivos" (hombre, 41 años, Cajamarca).

Tabla 3. Percepciones organizadas en categorías de adultos con problemas de salud mental autoidentificados de áreas rurales de Lima, sierra y selva del Perú: causalidad de los problemas de salud mental, situaciones que impiden su recuperación y recursos personales

\begin{tabular}{|c|c|}
\hline Percepciones & $(\%)$ \\
\hline \multicolumn{2}{|l|}{ Causas de los problemas de tipo emocional } \\
\hline Estresor familiar o conyugal & 24,6 \\
\hline Muerte de persona significativa & 15,7 \\
\hline Estresor social & 10,6 \\
\hline La salud & 10,2 \\
\hline Evento traumático & 10,2 \\
\hline Estresor económico & 8,5 \\
\hline La forma de ser & 4,7 \\
\hline Ninguno & 3,8 \\
\hline Soledad & 2,6 \\
\hline Enfermedad de persona significativa & 2,1 \\
\hline No responde & 7,0 \\
\hline Total & 100 \\
\hline \multicolumn{2}{|l|}{$\begin{array}{l}\text { Situaciones que impiden su recuperación del } \\
\text { problema de tipo emocional }\end{array}$} \\
\hline La forma de ser & 13,6 \\
\hline La salud & 11,9 \\
\hline Estresor económico & 9,8 \\
\hline Estresor familiar & 9,8 \\
\hline Estresor conyugal & 7,7 \\
\hline Estresor social & 6,8 \\
\hline Muerte de persona significativa & 5,5 \\
\hline Ninguno & 5,1 \\
\hline Evento traumático & 3,4 \\
\hline Soledad & 2,1 \\
\hline Enfermedad de persona significativa & 1,3 \\
\hline Otros & 0,9 \\
\hline No responde & 22,1 \\
\hline Total & 100 \\
\hline \multicolumn{2}{|c|}{$\begin{array}{l}\text { Recursos personales que ayudan a superar los } \\
\text { problemas de tipo emocional }\end{array}$} \\
\hline \multicolumn{2}{|c|}{ Recursos personales internos } \\
\hline Fortaleza/Fuerza voluntad & 19,1 \\
\hline Religión & 6,4 \\
\hline Alegría & 4,7 \\
\hline La salud & 2,1 \\
\hline \multicolumn{2}{|l|}{ Recursos personales externos } \\
\hline La familia & 11,1 \\
\hline Bienes inmateriales (profesión y trabajo) & 4,7 \\
\hline Dinero y bienes materiales & 0,4 \\
\hline No tiene & 12,8 \\
\hline No responde & 38,7 \\
\hline Total & 100 \\
\hline
\end{tabular}


$\sqrt{ }$ Desde que vi a unos hombres se peleaban con botella rota, me siento como asustada y también cuando el agente municipal golpeó al borracho, ahí me asustó mucho (mujer, 66 años, Loreto)

También se menciona eventos traumáticos $(10,2 \%)$, que representan circunstancias y vulnerabilidades del área rural, como son accidentes vehiculares, violencia, terrorismo, desastres naturales, etc. Los más mencionados fueron terremoto y terrorismo (en Lima y Ayacucho respectivamente).

¿Cuáles cree usted que son las causas o factores que dieron inicio a su problema de los nervios o de tipo emocional?

$\sqrt{ }$ Muerte violenta de mis familiares en choque (hombre, 46 años, Ancash).

$\sqrt{ }$ El terremoto de agosto pasado (...) Siempre me han asustado los sismos (...) Me he quedado traumada (mujer, 21 años, Ayacucho).

$\sqrt{ }$ Yo vi en Huamanguilla cómo los terroristas mataban a las autoridades, a las personas, a mis familias las han dejado heridas, yo me sentía asustado lloraba. (hombre, 40 años, Ayacucho).

En menor cantidad aparecen la falta de dinero o el poco dinero que se tiene o la falta de bienes materiales como casa, chacra, animales (estresor económico 8,5\%).

\section{SITUACIONES QUE IMPIDEN LA RECUPERACIÓN DE SU PROBLEMA DE TIPO EMOCIONAL}

Entre los aspectos más mencionados se encuentran su "forma de ser" $(13,6 \%)$ como la "debilidad", la inseguridad, desconfianza, la vergüenza, el nerviosismo y la exagerada preocupación, y la salud $(11,9 \%)$, es decir situaciones de orden interno o de personalidad. Como impedimentos externos se menciona el dinero, la pareja o la sociedad, entre otros. Otros impedimentos mencionados son el estresor económico y estresor familiar que alcanzan el 9,8\% (Tabla 3).

\section{RECURSOS PARA AFRONTAR LOS PROBLEMAS DE SALUD MENTAL}

\section{RECURSOS PERSONALES}

Menos de la mitad de los participantes (48,5\%) manifiestan tener recursos para superar su PSM. Como recursos internos mencionan en su mayoría $(19,1 \%)$ características que tienen que ver con la fortaleza, la valentía, la fuerza de voluntad, y su capacidad para enfrentar la adversidad. Esta es mencionada en su mayoría por personas alfabetas, con educación superior no universitaria y quienes hablan castellano. También mencionan en menor proporción la religión $(6,4 \%)$ (generalmente mencionado por personas de la selva, analfabetos y con menor grado de instrucción). Asimismo, se menciona la alegría, motivación y confianza para hacer cosas $(4,7 \%)$, y con menor frecuencia se señala a la salud $(2,1 \%)$ (Tabla 4$)$.

¿Qué recurso personal tiene usted que le ayude a superar su problema de tipo emocional?

$\sqrt{ }$ Tengo valor para seguir adelante, me da fuerza mi hijo, tengo fuerza para trabajar (mujer, 27 años, Cajamarca).

$\sqrt{ }$ Mi fe en Dios, creo que Él me puede ayudar. (hombre, 54 años, Ancash).

Con relación a los recursos externos, la familia es la más mencionada como recurso para enfrentar sus PSM $(11,1 \%)$, especialmente por personas de mayor edad. Aparecen también como recursos personales, pero en menor frecuencia, los bienes inmateriales, es decir el trabajo o la profesión (4,7\%), aunque son muy pocos los que tienen una profesión. Tampoco el dinero o los bienes materiales aparecen como recurso $(0,4 \%)$, reflejando, de esta manera, su condición de pobreza. En general, quienes consideran no tener recursos son los adultos mayores.

\section{RECURSOS COMUNITARIOS}

Más de la mitad de estos refieren que su comunidad no cuenta con recursos para ayudarlo en su problema

Tabla 4. Percepciones organizadas en categorías de adultos con problemas de salud mental autoidentificados de áreas rurales de Lima, sierra y selva del Perú: recurso comunitarios y expectativas de atención del establecimiento de salud

\begin{tabular}{lc}
\hline Percepciones & (\%) \\
\hline $\begin{array}{l}\text { Recursos de la comunidad para ayudarlo en su } \\
\text { problema }\end{array}$ \\
\hline La comunidad no tiene & 56,6 \\
\hline Apoyo emocional, moral, espiritual & 4,3 \\
\hline Apoyo material & 3,0 \\
\hline Programas sociales & 1,3 \\
\hline No responde & 34,8 \\
\hline Total & 100,00 \\
\hline Expectativas de atención en establecimiento de & \\
salud por su problema de tipo emocional & \\
Consejería & 24,3 \\
\hline Medicinas - Buena atención & 23,0 \\
\hline No sabe & 16,3 \\
\hline Buen trato respeto, comprensión, escucha & 13,6 \\
\hline Buen diagnóstico y tratamiento & 9,4 \\
\hline Nada & 3,0 \\
\hline Entrega de dinero, alimento y vestido & 2,6 \\
\hline No responde & 6,1 \\
\hline Total & 100 \\
\hline
\end{tabular}


emocional y solo $4 \%$ considera que existe apoyo emocional, moral o espiritual, como expresión de un soporte social pobre (Tabla 4). Los programas sociales son poco mencionados.

¿Con qué recursos cuenta su comunidad para ayudarlo en la recuperación de su problema de tipo emocional?

$\sqrt{ }$ La posta no hace nada, no da charlas ni nos orienta, ni a los jóvenes (hombre, 70 años, Ancash).

$\sqrt{ }$ No, porque la gente aquí es muy pobre (hombre, 34 años, Ayacucho).

$\sqrt{ }$ Aquí no hay medicinas, tengo que irme lejos cuando estoy mal del estómago (hombre, 58 años, Lima).

\section{EXPECTATIVAS DE ATENCIÓN EN LOS ESTABLECIMIENTOS DE SALUD}

El $24,3 \%$ de las respuestas de los participantes tienen como expectativa de atención recibir consejos y orientación. Esto, generalmente, es mencionado en menor cantidad por los participantes más jóvenes y quechua hablantes. El $23,0 \%$ de las respuestas hacen referencia a medicina y buena atención. El 13,6\% de las respuestas hacen mención al buen trato que esperan recibir de parte de los profesionales, lo que implica que los escuchen, que sean amables, que les brinden confianza, cariño y comprensión. Debemos resaltar que un $16,3 \%$ respondió que no sabe qué tipo de atención esperar.

¿Qué atención espera recibir en un establecimiento de salud por su problema de tipo emocional?

$\sqrt{ }$ Buena atención y me calme la preocupación hablándome y orientándome, regresando a mi casa contenta y feliz con la explicación que me da (mujer, 23 años, Ayacucho).

$\checkmark$ Que me brinden medicamentos para recuperarme (hombre, 67 años, Ancash).

$\sqrt{ }$ Que atiendan siempre, porque para cerrado (hombre, 78 años, Ayacucho).

$\sqrt{ }$ Que nos entiendan y nos escuchen (mujer de 54 años, Ayacucho).

$\sqrt{ }$ Amabilidad, confianza, buen trato (mujer, 25 años, Ancash).

$\sqrt{ }$ Con cariño y con apoyo y sanarme y no matarme como a otros (mujer, 33 años, Ancash).

\section{DISCUSIÓN}

Se ha presentado algunas percepciones que sobre sus PSM tienen adultos de regiones rurales del Perú, encontrándose particularidades que obedecen al contexto de social, cultural y de pobreza que caracteriza estas regiones. A nuestro entender, la presente investigación es el primer estudio que integra un enfoque cualitativo a un estudio de tipo epidemiológico sobre la perspectiva de la persona que padece PSM.

Entre las causas de los PSM más frecuentemente citadas resaltan los problemas conyugales y familiares, especialmente en las mujeres. Coincide con el reporte de una mayor prevalencia de violencia doméstica al interior del país en comparación a Lima Metropolitana ${ }^{(11,12,13,22)}$. Esto podría explicarse porque en el área rural la mujer asumiría con menos cuestionamiento los roles impuestos por el entorno social ${ }^{(23)}$, lo que la colocaría en mayor riesgo, debido a la importancia de las relaciones familiares coherentes y cohesionadas como factor protector de la salud mental ${ }^{(24)}$. Se señala también a la muerte como causa de estos problemas, lo que se aplicará a que, en las zonas rurales, la pérdida de uno de sus miembros implica la pérdida física y la pérdida de una persona útil para el trabajo ${ }^{(10)}$. Se menciona también a la salud física como un elemento causal importante, lo que concordaría con hallazgos que vinculan a la salud física como mediador de la felicidad ${ }^{(25)}$. Debido a las brechas de atención de salud que subsisten en el área rural, se convierte en un factor de vulnerabilidad para la salud mental de estas poblaciones.

Con respecto a las situaciones que impiden la recuperación del PSM resaltan los problemas familiares. También se menciona "la forma de ser" como una forma de problemas de personalidad. Asimismo, el mismo PSM se menciona como limitante para la recuperación de la persona lo que refuerza la necesidad de mayor intervención de recursos humanos psicológicos en este campo de la salud en estas áreas. Esto concuerda con las expectativas que tiene la población del establecimiento de salud, ya que el tipo de atención más frecuentemente esperada es la consejería y luego la atención médica.

Como recursos personales internos para superar sus PSM resalta aspectos de salud positiva como la fortaleza, fuerza de voluntad o la alegría, reportados como elementos preventivo promocionales de la salud mental (26). La religión aparece en segundo lugar, coincidiendo con un estudio reciente que encontró que la asistencia frecuente a servicios religiosos estaba asociada significativamente con tasas menores de suicidio, y sugiriendo que la religión y la espiritualidad pueden ser un recurso subapreciado por psiquiatras y clínicos y que podría estimularse como una forma de participación social significativa (27). La familia no aparece como un recurso frecuente para superar PSM, probablemente debido a los escasos recursos del grupo familiar. También debemos notar que existen personas que consideran no tener ningún recurso personal, información importante para un adecuado plan de tratamiento y soporte social personalizado.

Un área preocupante corresponde a la percepción de falta de recursos comunitarios para superar sus PSM. Estos hallazgos contrastan con un estudio recientemente 
de Selamu et al. quienes encontraron en una comunidad rural etíope abundantes recursos comunitarios, tanto religiosos como de medicina tradicional y asociaciones sociales que podrían utilizarse para fomentar y mejorar la atención en la salud mental y proteger los derechos humanos ${ }^{(28)}$. Las comunidades estudiadas contarían con escasos recursos, lo que pondría a estas personas en una desventaja mayor. Por otro lado, cerca de una quinta parte de las personas no saben qué esperar del establecimiento de salud, o no esperan nada de él, lo que involucra una demanda desinformada de sus derechos en el área de la salud mental.

\section{CONCLUSIONES}

Existen muchos aspectos de la cultura detrás de la salud, que son pertinentes para el diseño de políticas de salud pública. Resaltan los problemas de la familia relacionados con temas como violencia intrafamiliar y relaciones interpersonales en el marco de la escasez de recursos y problemas de género. Hay una importante falta de apoyo comunitario y las personas tienen expectativas de una atención clínica de consejería, más cercana y personal. El presente estudio pone de manifiesto el círculo vicioso entre las carencias económicas y la salud mental donde confluyen las limitaciones en los recursos comunitarios de salud, y una débil estructura y red social que no acoge importantemente estos problemas de salud, y que es importante para la seguridad y bienestar ${ }^{29)}$. Urge también el rescate de los valores comunales de ayuda mutua y colectiva, así como de los lazos de solidaridad presentes en la comunidad. Consideramos que el enfoque narrativo en este estudio epidemiológico nos pone en contacto directo con las personas y con sus necesidades, y formas de evaluar la realidad en el marco de sus PSM, que lo convierte en una herramienta importante de tipo cualitativo en el enfoque de una medicina centrada en la persona y la comunidad.

Contribución de los autores: JES ha participado en la concepción de la investigación, diseño del artículo, análisis de datos, redacción y aprobación de la versión final; VU ha participado en el análisis de datos, redacción y aprobación de la versión final.

Conflictos de interés: el autor no declara ningún conflicto de interés.

Fuentes de financiamiento: el estudio original recibió financiamiento del Instituto Nacional de Salud Mental "Honorio Delgado - Hideyo Noguchi"

\section{REFERENCIAS BIBLIOGRÁFICAS}

1 Starfield B. The hidden inequity in health care. Int J of Equity in Health. 2011; (10:15 doi:10.1186/1475-9276$10-15)$

2 Rutz W, Fernandez M, Trivedi J. Social Perspectives on Psychiatry for the Person. Int J Person Centered Medicine. 2011;1140-142.

3 Hughes JC, Bamford C, May C. Types of centeredness in health care: themes and concepts. Med Health care and Philos. 2008; 11:455-463.

4 Korkeila J. Measuring aspects of mental health. Helsinky: STAKES, 2000.

5 Stewart M. Towards a global definition of patient centered care. The patient should be the judge of patient centred care. BMJ. 2001; 322:444-5.

6 Phillips, J. Idiographic formulations, Symbols, Narratives, Context and Meaning. Psychpathology. 2005; 38:180-184.

7 IGDA Workgroup, WPA. IGDA 8: Idiographic (personalised) diagnostic formulation. British Journal of Psychiatry. 2003; 182 (suppl.45), 55-57.

8 Asociación Psiquiátrica de América Latina, Sección de Diagnóstico y Clasificación. Guía Latinoamericana de Diagnóstico Psiquiátrico. Guadalajara:
Asociación Psiquiátrica de América Latina, 2004

9 IGDA Workgroup, WPA. IGDA. Introduction. Br J Psychiatry. 2003; 182 (supp. 45): s37-s39.

10 Yancari J. Crisis y pobreza rural en América Latina: el caso de Perú. Documento de trabajo No41. Lima: IEP, Centro Latinoamericano para el desarrollo rural; 2009.

11 Instituto Nacional de Salud Mental. Estudio Epidemiológico de Salud Mental en Lima Rural 2007. Anales de Salud Mental. 2008; XXIV (1 y 2): 1-247.

12 Instituto Nacional de Salud Mental. Estudio Epidemiológico de Salud Mental en la Sierra Rural Rural 2008. Informe General. Anales de Salud Mental. 2009; XXV (1 y 2): 1-318.

13 Instituto Nacional de Salud Mental. Estudio Epidemiológico de Salud Mental en la Selva Rural Rural 2009. Informe General. Anales de Salud Mental. 2012; XXVIII (Suplemento 2): $1-343$

14 Instituto Nacional de Salud Mental. Confiabilidad y validez de los cuestionarios de salud mental de Lima y de la Selva Peruana. Anales de Salud Mental. 2009; XXV (Supl. 1): 1-259.
15 IGDA Workgroup, WPA. IGDA. 8. Idiographic (personalized) diagnostic formulation. Br J Psychiatry. 2003; 182 (supp. 45): s55-s57

16 Sección de Diagnóstico y Clasificación de la Asociación Psiquiátrica de América Latina. Guía Latinoamericana de Diagnóstico Psiquiátrico. Guadalajara: Asociación Psiquiátrica de América Latina; 2004.

17 Sheehan DV, Lecrubier Y, Sheehan $\mathrm{KH}$, Amorim P, Janavs J, Weiller $\mathrm{E}$, et al. The Mini-International Neuropsychiatric Interview (M.I.N.I.): The Development and Validation of a Structured Diagnostic Psychiatric Interview for DSM-IV and ICD-10. J Clin Psychiatry. 1998; 59 Suppl. 20: 22-33.

18 INEI. Manual de la Encuestadora de la Encuesta Nacional de Hogares 2000 IV trimestre Oct 2000.

19 Feres JC, Macero X. Enfoques para la medición de la pobreza. Breve revisión de la literatura. Santiago de Chile: UN; 2001 Available at: http://www.eclac. org/publicaciones/Estadisticas/9/ lcl1479/lcl1479e.pdf. Accessed Nov 3, 2005.

20 Valles MS. Introducción a la Metodología del análisis cualitativo: 
Panorámica de procedimientos y técnicas. En: Técnicas cualitativas de investigación social. Reflexión metodológica y práctica profesional. Madrid: Síntesis Psicológica; 1997.

21 Rodríguez G, Gil J, García E. Metodología de la investigación cualitativa. Málaga: Ediciones Aljibe; 1996.

22 Instituto Nacional de Salud Mental "Honorio Delgado - Hideyo Noguchi”. Estudio Epidemiológico de Salud Mental en Lima Metropolitana y Callao- Replicación 2012. Informe General. Anales de Salud Mental. 2013; XXIX (Supl 1): 1-392

23 Raguz, M. Construcciones sociales y psicológicas de mujer, hombre, femineidad, masculinidad y género en diversos grupos poblacionales. Lima: Pontificia Universidad Católica del Perú; 1995
24 Rivera FI, Guarnaccia PJ, MulvaneyDay N, Lin JY, Torres M, Alegría M. Family Cohesion and its Relationship to Psychological Distress among Latino Groups. Hisp J Behav Sci. 2008; 30(3): 357-378. doi: $10.1177 / 0739986308318713$.

25 Alarcón R. Fuentes de felicidad: ¿Qué hace feliz a la gente? Revista de Psicología de la PUCP. 2002; 20(2):170-196.

26 Fredrickson BL, Tugade MM, Waugh CE, Larkin GR. What Good Are Positive Emotions in Crises? A Prospective Study of Resilience and Emotions Following the Terrorist Attacks on the United States on September 11th, 2001. J Pers Soc Psychol. 2003; 84(2): 365-376.

27 VanderWeele TJ,LiS,Tsai AC, Kawachi I. Association Between Religious Service Attendance and Lower Suicide Rates Among US Women.
JAMA Psychiatry. doi:10.1001/ jamapsychiatry.2016.1243. Published online June 29, 2016.

28 Selamu M, Asher L, Hanlon C, Medhin G, Hailemariam M, Patel V, Thornicroft G, Fekadu A. Beyond the biomedical: community resources for mental health care in rural Ethiopia. PLoS One. 2015 May 11;10(5):e0126666. doi: 10.1371/ journal.pone.0126666. eCollection 2015.

29 Pedersen D. Salud mental de la comunidad. Lima: IPAZ, McGill, UPCH; 2007.

Correspondencia: Javier Saavedra Dirección: Jr. Eloy Espinoza Saldaña 709, San Martín de Porras, Lima, Perú Teléfono: +5116149201

Correoelectrónico: saavedra@terra.com.pe

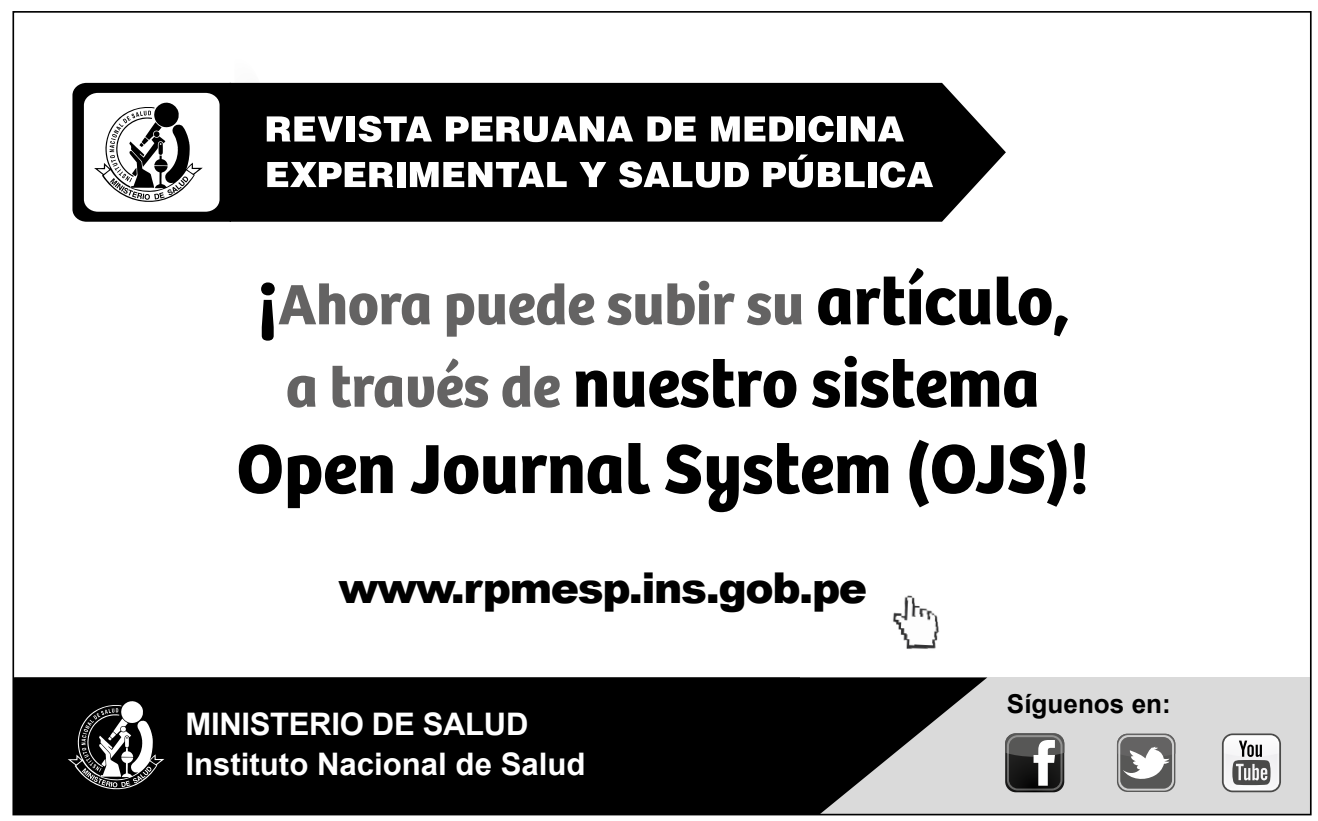

